\title{
Perfect Simulation of the Hard Disks Model by Partial Rejection Sampling
}

\section{Heng Guo}

School of Informatics, University of Edinburgh, Informatics Forum, Edinburgh, EH8 9AB, United Kingdom.

hguo@inf.ed.ac.uk

(D) https://orcid.org/0000-0001-8199-5596

\section{Mark Jerrum ${ }^{1}$}

School of Mathematical Sciences, Queen Mary, University of London, Mile End Road, London, E1 4NS, United Kingdom.

m.jerrum@qmul.ac.uk

(D) https://orcid.org/0000-0003-0863-7279

\begin{abstract}
We present a perfect simulation of the hard disks model via the partial rejection sampling method. Provided the density of disks is not too high, the method produces exact samples in $O(\log n)$ rounds, where $n$ is the expected number of disks. The method extends easily to the hard spheres model in $d>2$ dimensions. In order to apply the partial rejection method to this continuous setting, we provide an alternative perspective of its correctness and run-time analysis that is valid for general state spaces.
\end{abstract}

2012 ACM Subject Classification Theory of computation $\rightarrow$ Randomness, geometry and discrete structures

Keywords and phrases Hard disks model, Sampling, Markov chains

Digital Object Identifier 10.4230/LIPIcs.ICALP.2018.69

Related Version Also available at https://arxiv.org/abs/1801.07342.

Acknowledgements We thank Mark Huber and Will Perkins for inspiring conversations and bringing the hard disks model to our attention.

\section{Introduction}

The hard disks model is one of the simplest gas models in statistical physics. Its configurations are non-overlapping disks of uniform radius $r$ in a bounded region of $\mathbb{R}^{2}$. For convenience, in this paper, we take this region to be the unit square $[0,1]^{2}$. This model was precisely the one studied by Metropolis et al. [12], in their pioneering work on the Markov chain Monte Carlo (MCMC) method. They used Los Alamos' MANIAC computer to simulate a system with 224 disks.

There are two variants of this model. To obtain the canonical ensemble, we fix the number (or equivalently, density) of disks and decree that all configurations are "equally likely", subject only to the disks not overlapping. In the grand canonical ensemble, we fix the "average" number of disks. To be more specific, centers of the disks are distributed 1 The work described here was supported by the EPSRC research grant EP/N004221/1 "Algorithms that
Count".

(c) (i) $($ Heng Guo and Mark Jerrum;

licensed under Creative Commons License CC-BY

45th International Colloquium on Automata, Languages, and Programming (ICALP 2018). Editors: Ioannis Chatzigiannakis, Christos Kaklamanis, Dániel Marx, and Donald Sannella; Article No. 69; pp. 69:1-69:10

Leibniz International Proceedings in Informatics

LIPICS Schloss Dagstuhl - Leibniz-Zentrum für Informatik, Dagstuhl Publishing, Germany 
according to a Poisson point process of intensity $\lambda_{r}=\lambda /\left(\pi r^{2}\right)$, conditioned on the disks being non-overlapping. The hard disks model, and its higher dimensional generalization (called the hard spheres model) are also related to the optimal sphere packing density [4, 18, 2]. See $[6,1]$ and references therein for more details. See also [11] for the physics perspective.

Our main aim in this work is to describe and analyse a very simple algorithm for exactly sampling from the grand canonical ensemble, based on the partial rejection sampling paradigm introduced by Guo, Jerrum and Liu [3].

More precisely, the challenge is the following: produce a realisation $P \subset[0,1]^{2}$ of a Poisson point process of intensity $\lambda_{r}$ in the unit square, conditioned on the event that no pair of points in $P$ are closer than $2 r$ in Euclidean distance. We refer to this target measure as the hard disks distribution. It describes an arrangement of open disks of radius $r$ with centres in $[0,1]^{2}$ that are not allowed to overlap, but which otherwise do not interact. It is a special case of the Strauss process [17]. Note that, although the disks do not overlap each other, they may extend beyond the boundary of the unit square. Also, the intensity of the underlying Poisson process is normalised so that the expected number of points of $P$ lying in a disk of radius $r$ is $\lambda$. This normalisation gives us sensible asymptotics as the radius of the disks tends to zero (equivalently, the number of disks tends to infinity).

Classical rejection sampling applied to this problem yields the following algorithm: repeatedly sample a realisation $P$ of the Poisson process of intensity $\lambda$ in the unit square until $P$ satisfies the condition that no two points are closer than $2 r$, and return $P$. Unfortunately, for every $\lambda>0$, however small, the expected number of unsuccessful trials using this approach increases exponentially in $r^{-1}$, as $r \rightarrow 0$. Partial rejection sampling [3] requires only a subset of $P$ to be resampled at each iteration. Algorithm 1 below arises from a routine application of the paradigm to the problem at hand.

The original partial rejection method [3] and its analysis are tailored for the discrete case. In this paper we provide an alternative view on the correctness of the method, which is also valid in the continuous setting. In other words, as with classical rejection sampling, Algorithm 1 terminates with probability 1, producing a realisation of the exact hard disks distribution. In contrast to classical rejection sampling, the expected number of iterations (resampling steps) is now asymptotically $O\left(\log \left(r^{-1}\right)\right)$ as $r \rightarrow 0$, provided $\lambda$ is not too large. We prove that rapid termination occurs when $\lambda<0.21027$. This analysis is not tight, and experiments suggests that the actual threshold for rapid termination is around $\lambda \approx 0.51 .^{2}$ The experimental advantage of partial rejections is its simple termination rule, unlike MCMC, where it is difficult to determine how long the algorithm should run.

The method extends naturally to the hard spheres model in $d>2$ dimensions. Here, the desired distribution is a Poisson point process in $[0,1]^{d}$ conditioned on no pair of points being closer than $2 r$. The natural normalisation for the intensity of the Poisson process in $d$ dimensions is $\lambda_{r, d}=\lambda /\left(v_{d} r^{d}\right)$, where $v_{d}$ is the volume of a ball of radius 1 in $\mathbb{R}^{d}$. With this convention, we prove that rapid termination occurs in $d$ dimensions provided $\lambda<2^{-\left(d+\frac{1}{2}\right)}$.

The expected packing density $\alpha(\lambda)$ or simply $\alpha$ for this model is the expected total volume of balls. (Note that, neglecting boundary effects, $\alpha$ is the proportion of the unit cube occupied by balls.) The quantity $\alpha(\lambda)$ grows monotonically with $\lambda$, but intuitively we expect its rate of growth to slow down dramatically as the balls pack more tightly. The connection between expected packing density $\alpha$ and intensity $\lambda$ has recently been thoroughly explored by Jenssen, Joos and Perkins [6]. Using their results, we show that partial rejection sampling

2 The physics prediction of phase transitions, for the canonical ensemble in two dimensions, is $\approx 0.7$. This threshold is more related to the expected packing density $\alpha(\lambda)$ discussed below. 


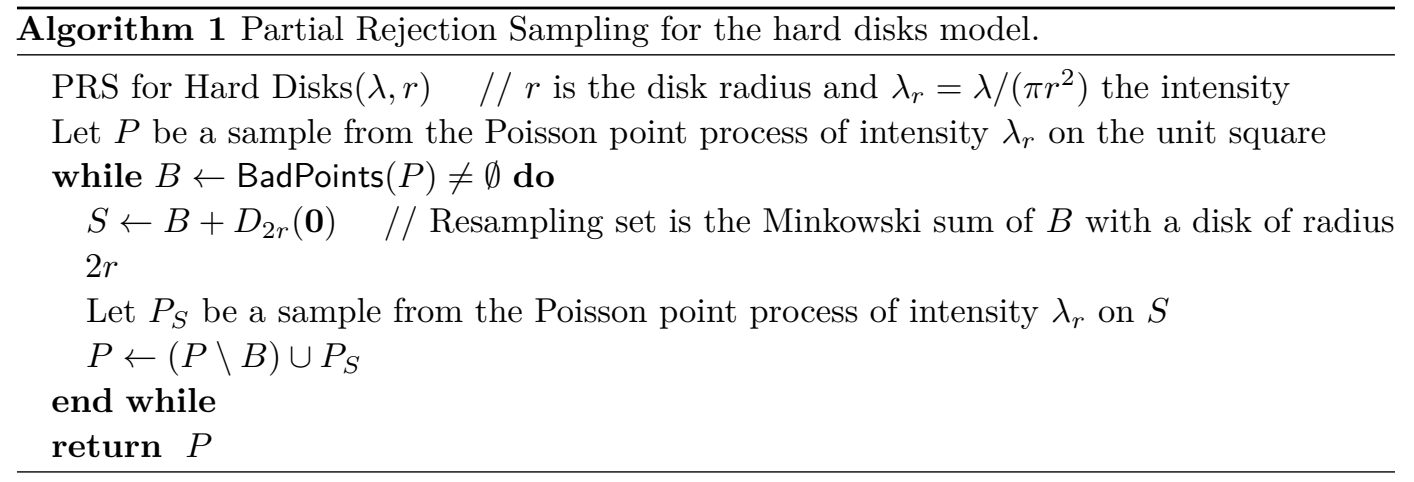

can achieve expected packing density $\Omega\left(2^{-d}\right)$ while retaining runtime $O\left(\log \left(r^{-1}\right)\right)$. Although sphere packings of density $\Omega\left(d 2^{-d}\right)$ have been proved to exist, there is no polynomial-time sampler that provably achieves packing density beyond $O\left(2^{-d}\right)$, as far as we are aware.

Other approaches to exact sampling include Coupling From The Past (CFTP), which was adapted to point processes by Kendall [8] and Kendall and Møller [9]. Recently, Moka, Juneja and Mandjes [13] proposed an algorithm based on rejection and importance sampling. Although this algorithm, like ours, is based on rejection sampling, it does not share our asymptotic performance guarantee. Indeed, its running time appears to grow exponentially as the number of disks goes to infinity, with the density of disks held constant. See also $[6,14]$ for rigorous bounds on packing densities of grand canonical ensembles.

Approximate sampling via Markov chain simulation has been studied by Kannan, Mahoney and Montenegro [7] and Hayes and Moore [5] in the context of the canonical ensemble, where the number of disks in a configuration is fixed. The best rigorous density bound to guarantee rapid mixing is below $\approx 0.154[5]$. It is should be noted that this is not directly comparable with our $\lambda<0.21027$ due to the difference in models. To obtain canonical ensembles, we could use Algorithm 1 and further condition on the number of desired disks. However, the only rigorous guarantee of this approach, via [6], is $\alpha(0.21027)>0.0887$.

\section{The sampling algorithm}

The following notation will be used throughout. If $P$ is a finite subset of $[0,1]^{2}$ then

$$
\operatorname{BadPairs}(P)=\{\{x, y\}: x, y \in P \wedge x \neq y \wedge\|x-y\|<2 r\} \text {, }
$$

where $\|\cdot\|$ denotes Euclidean norm, and

$$
\operatorname{BadPoints}(P)=\bigcup \operatorname{BadPairs}(P)
$$

The open disk of radius $r$ with centre $x \in[0,1]^{2}$ is denoted by $D_{r}(x)$. The finite set $\Pi \subset[0,1]^{2}$ always denotes a realisation of the Poisson point process of intensity $\lambda_{r}$ on $[0,1]^{2}$. For a random variable $X$ and event $\mathcal{E}$ we use $\mathcal{D}(X)$ to denote the distribution (law) of $X$, and $\mathcal{D}(X \mid \mathcal{E})$ the distribution of $X$ conditioned on $\mathcal{E}$ occurring. Thus, $\mathcal{D}(\Pi \mid \operatorname{BadPoints}(\Pi)=\emptyset)$ is the hard disks distribution that we are interested in.

Our goal is to analyse the correctness and running time of a sampling algorithm for the hard disks model (see Algorithm 1 below), specifically to determine the largest value of $\lambda$ for which it terminates quickly, i.e., in $O\left(\log r^{-1}\right)$ iterations. The algorithm is an example application of "Partial Rejection Sampling" [3], adapted to the continuous state space setting. 


\section{Correctness}

Let $B$ be any finite subset of $[0,1]^{2}$. We say that $B$ is a feasible set of bad points if $\operatorname{BadPoints}(B)=B$; this is equivalent to saying that there is a finite subset $R \subset[0,1]^{2}$ with $B=\operatorname{BadPoints}(R)$. The key to establishing correctness of Algorithm 1 is the following loop invariant:

$$
\mathcal{D}(P \mid \operatorname{BadPoints}(P)=B)=\mathcal{D}(\Pi \mid \operatorname{BadPoints}(\Pi)=B),
$$

for every feasible set $B$, where $P$ is any intermediate set of points during the execution of the algorithm, and $\Pi$ is a realisation of the Poisson point process. Let us consider what the right hand side means operationally. Let $S=B+D_{2 r}(\mathbf{0})$. (This is the resampling set used by the algorithm.) Let $Q$ be a sample from the distribution $\mathcal{D}(\Pi \mid \operatorname{BadPoints}(\Pi)=B)$. The only points in $Q$ that lie inside $S$ are the points in $B$. (Any extra points would create more bad pairs than there actually are.) Thus $Q \cap S=B$. Outside of $S$ there are no bad pairs; thus $Q \cap \bar{S}$ is a sample from the hard disks distribution on $\bar{S}=[0,1]^{2} \backslash S$. Note that, setting $B=\emptyset$, we see that $\mathcal{D}(\Pi \mid \operatorname{BadPoints}(\Pi)=\emptyset)$ is just the hard disks distribution on $[0,1]^{2}$.

- Theorem 1. Algorithm 1 is correct: conditional on halting, Algorithm 1 produces a sample from the hard disks distribution with intensity $\lambda_{r}=\lambda /\left(\pi r^{2}\right)$.

Theorem 1 follows from Lemma 2 below.

Let $T$ (a random variable) be the number of iterations of the while-loop. On each iteration, the while loop terminates with probability bounded away from 0 ; thus $T$ is finite with probability 1. (Indeed, $T$ has finite expectation.) Let $P_{t}$, for $1 \leq t \leq T$, be the point set $P$ after $t$ iterations of the loop, and $P_{0}$ be the initial value of $P$ (which is just a realisation of the Poisson point process on $\left.[0,1]^{2}\right)$. We say that $B_{0}, B_{1}, \ldots, B_{t} \subset[0,1]^{2}$ is a feasible sequence of (finite) point sets if there exists a run of $\operatorname{Algorithm} 1$ with $\operatorname{BadPoints}\left(P_{0}\right)=$ $B_{0}, \ldots$, BadPoints $\left(P_{t}\right)=B_{t}$.

- Lemma 2. Let $B_{0}, B_{1}, \ldots, B_{t} \subset[0,1]^{2}$ be a feasible sequence. Then

$$
\begin{aligned}
\mathcal{D}\left(P_{t} \mid \operatorname{BadPoints}\left(P_{0}\right)=B_{0} \wedge \cdots \wedge \operatorname{BadPoints}\left(P_{t}\right)=B_{t}\right) & =\mathcal{D}\left(P_{t} \mid \operatorname{BadPoints}\left(P_{t}\right)=B_{t}\right) \\
& =\mathcal{D}\left(\Pi \mid \operatorname{BadPoints}(\Pi)=B_{t}\right)
\end{aligned}
$$

Proof. We prove the result by induction on $t$. The base case, $t=0$, holds by construction: $P_{0}$ is just a realisation of the Poisson point process on $[0,1]^{2}$. Our induction hypothesis is

$$
\mathcal{D}\left(P_{t} \mid \operatorname{BadPoints}\left(P_{0}\right)=B_{0} \wedge \cdots \wedge \operatorname{BadPoints}\left(P_{t}\right)=B_{t}\right)=\mathcal{D}\left(\Pi \mid \text { BadPoints }(\Pi)=B_{t}\right),
$$

for every feasible sequence $B_{0}, \ldots, B_{t}$. Extend the feasible sequence to $B_{t+1}$. For the inductive step, we assume (1) and aim to derive

$$
\begin{aligned}
\mathcal{D}\left(P_{t+1} \mid \operatorname{BadPoints}\left(P_{0}\right)=B_{0} \wedge \cdots \wedge \operatorname{BadPoints}\left(P_{t+1}\right)=B_{t+1}\right) \\
=\mathcal{D}\left(\Pi \mid \operatorname{BadPoints}(\Pi)=B_{t+1}\right) .
\end{aligned}
$$

The resampling set on iteration $t+1$ is $S=B_{t}+D_{2 r}(\mathbf{0})$. As a first step we argue below that

$$
\mathcal{D}\left(P_{t+1} \mid \operatorname{BadPoints}\left(P_{0}\right)=B_{0} \wedge \cdots \wedge \operatorname{BadPoints}\left(P_{t}\right)=B_{t}\right)=\mathcal{D}\left(\Pi \mid \operatorname{BadPairs}(\Pi) \cap \bar{S}^{(2)}=\emptyset\right),
$$

where $\bar{S}^{(2)}$ denotes the set of unordered pairs of elements from $\bar{S}$. We have noted that (1) implies that, outside of the resampling set $S$, the point set $P_{t}$ is a realisation of the 
hard disks distribution. Also, the algorithm does not resample points outside of $S$. Thus $P_{t+1} \cap \bar{S}=P_{t} \cap \bar{S}$ is Poisson distributed, conditioned on there being no bad pairs. Inside $S$, resampling has left behind a fresh Poisson point process $P_{t+1} \cap S$. These considerations give (3).

Next, we condition on $B_{t+1}$ :

$$
\begin{aligned}
\mathcal{D}\left(P_{t+1} \mid \operatorname{BadPoints}\left(P_{0}\right)=\right. & \left.B_{0} \wedge \cdots \wedge \operatorname{BadPoints}\left(P_{t}\right)=B_{t} \wedge \operatorname{BadPoints}\left(P_{t+1}\right)=B_{t+1}\right) \\
& =\mathcal{D}\left(\Pi \mid \operatorname{BadPairs}(\Pi) \cap \bar{S}^{(2)}=\emptyset \wedge \operatorname{BadPoints}(\Pi)=B_{t+1}\right) .
\end{aligned}
$$

Since $B_{t+1}$ contains no bad pairs with both endpoints in $\bar{S}$, the event BadPoints $(\Pi)=B_{t+1}$ entails the event BadPairs $(\Pi) \cap \bar{S}^{(2)}=\emptyset$. Thus, we have

$$
\begin{array}{r}
\mathcal{D}\left(P_{t+1} \mid \operatorname{BadPoints}\left(P_{0}\right)=B_{0} \wedge \cdots \wedge \operatorname{BadPoints}\left(P_{t}\right)=B_{t} \wedge \operatorname{BadPoints}\left(P_{t+1}\right)=B_{t+1}\right) \\
=\mathcal{D}\left(\Pi \mid \operatorname{BadPoints}\left(P_{t+1}\right)=B_{t+1}\right)
\end{array}
$$

The right hand side of this equation does not involve $B_{0}, \ldots, B_{t}$, and so

$$
\mathcal{D}\left(P_{t+1} \mid \text { BadPoints }\left(P_{t+1}\right)=B_{t+1}\right)=\mathcal{D}\left(\Pi \mid \text { BadPoints }(\Pi)=B_{t+1}\right) .
$$

This completes the induction step (2) and the proof.

Proof of Theorem 1. As we observed earlier, T, the number of iterations of the while-loop, is finite with probability 1 . By Lemma 2 , noting that $B_{T}=\emptyset$,

$$
\mathcal{D}\left(P_{T}\right)=\mathcal{D}(\Pi \mid \text { BadPoints }(\Pi)=\emptyset) .
$$

In other words, at termination, Algorithm 1 produces a realisation of the hard disks distribution on $[0,1]^{2}$.

\section{Run-time analysis}

We consider how the number of "bad events", i.e., the cardinality of the set BadPairs $\left(P_{t}\right)$, evolves with time. As usual $\Pi$ denotes a realisation of the Poisson point process of intensity $\lambda_{r}$. Also denote by $\Delta$ a realisation of the hard disks process of the same intensity. We need the following stochastic domination result.

- Lemma 3. The hard disks distribution is stochastically dominated by the Poisson point process with the same intensity. That is, we can construct a joint sample space for $\Pi$ and $\Delta$ such that $\Delta \subseteq \Pi$.

Holley's criterion is a useful test for stochastic domination, but it is not of direct use to us in the proof of Lemma 3, because it applies only to finite state spaces. Fortunately, Preston [15, Theorem 9.1], has derived a version of Holley's criterion that fits our situation. We will mostly follow Preston's notation, except that, to save confusion, we will use $P$ and $Q$, rather than $x$ and $y$, to denote finite sets of points. In order to state his result, we need some notation. In our application, $\widetilde{\omega}_{n}$ is the distribution on $\left([0,1]^{2}\right)^{(n)}$ obtained by sampling $n$ points independently and uniformly at random from $[0,1]^{2}$, and regarding the points as indistinguishable; furthermore, $\widetilde{\omega}=\sum_{n=0}^{\infty} \widetilde{\omega}_{n} / n$ !. (For consistency with Preston, we have left $\widetilde{\omega}$ unnormalised. If we had made it into a probability distribution by division by $e$, then $\widetilde{\omega}$ could be thought of as follows: sample an integer $k$ from the Poisson distribution with mean 1 , and then pick $k$ (unlabelled) points uniformly and independently.) Denote by $\Omega$ 
the set of all finite subsets of $[0,1]^{2}$, and by $\mathcal{F}$ the set of non-negative measurable functions $\Omega \rightarrow \mathbb{R}$ satisfying

$$
\int f d \widetilde{\omega}=1
$$

and

$$
f(P)=0 \text { and } P \subseteq Q \text { implies } f(Q)=0 .
$$

(See Preston [15, Section 9] for detailed formal definitions of the concepts here.)

- Lemma 4 (Theorem 9.1 of [15]). Let $f_{1}, f_{2} \in \mathcal{F}$ and suppose that

$$
\frac{f_{1}(P+\xi)}{f_{1}(P)} \geq \frac{f_{2}(Q+\xi)}{f_{2}(Q)}, \quad \text { for all } Q \subseteq P \in \Omega \text { and } \xi \in[0,1]^{2} \backslash P
$$

(where, by convention, $0 / 0=0$ ). Then, for all bounded, measurable, non-decreasing functions $g: \Omega \rightarrow \mathbb{R}$

$$
\int g f_{1} d \widetilde{\omega} \geq \int g f_{2} d \widetilde{\omega}
$$

i.e., if $\mu_{i}$ is the probability measure having density $f_{i}$ with respect to $\widetilde{\omega}$, then $\mu_{1}$ stochastically dominates $\mu_{2}$.

Proof of Lemma 3. We set

$$
f_{1}(P)=C_{1} \lambda_{r}^{|P|}
$$

and

$$
f_{2}(P)= \begin{cases}C_{2} \lambda_{r}^{|P|}, & \text { if } \operatorname{BadPairs}(P)=\emptyset ; \\ 0, & \text { otherwise. }\end{cases}
$$

The normalising constants $C_{1}$ and $C_{2}$ are chosen so that both $f=f_{1}$ and $f=f_{2}$ satisfy (4). (There is an explicit expression for $C_{1}$, namely $C_{1}=\exp \left(-\lambda_{r}\right)$, but not for $C_{2}$.) Notice that both $f_{1}$ and $f_{2}$ also satisfy (5). Notice also that the probability measures $\mu_{1}$ and $\mu_{2}$ of the Poisson point process and the hard disks process have densities $f_{1}$ and $f_{2}$ with respect to $\widetilde{\omega}$. The premise (6) of Lemma 4 holds, since the left-hand side is always $\lambda_{r}$ and the right-hand side is either $\lambda_{r}$ or 0 . The conclusion is that $\mu_{1}$ dominates $\mu_{2}$. Strassen's Theorem [10, 16], allows us to conclude the existence of a coupling of $\Pi$ and $\Delta$ as advertised in the statement of the lemma (except, possibly, on a set of measure zero).

- Lemma 5. There is a bound $\bar{\lambda}>0$ such that the expected number of iterations of the while-loop in Algorithm 1 is $O\left(\log r^{-1}\right)$ when $\lambda<\bar{\lambda}$.

Proof. First observe that $\operatorname{BadPairs}(P)$ determines $\operatorname{BadPoints}(P)$ and vice versa. So conditioning on the set $\operatorname{BadPairs}(P)$ is equivalent to conditioning on $\operatorname{BadPoints}(P)$.

Introduce random variables $Z_{t}=\left|\operatorname{BadPairs}\left(P_{t}\right)\right|$, for $1 \leq t \leq T$. Our strategy is to show that

$$
\mathbb{E}\left(Z_{t+1} \mid Z_{0}, \ldots, Z_{t}\right) \leq \alpha^{-1} Z_{t}
$$

for some $\alpha>1$. Then $Z_{0}, \alpha Z_{1}, \alpha^{2} Z_{2}, \alpha^{3} Z_{3}, \ldots$ is a supermartingale (with the convention that $Z_{t}=0$ for all $t>T$ ). Therefore, $\mathbb{E} Z_{t} \leq \alpha^{-t} \mathbb{E} Z_{0} \leq \frac{1}{2} \lambda_{r}^{2} \alpha^{-t}$. Here, we have used the fact that $\left|P_{0}\right|$ is a Poisson random variable with expectation $\lambda_{r}$, and

$$
Z_{0}=\left|\operatorname{BadPairs}\left(P_{0}\right)\right| \leq \frac{1}{2}\left|P_{0}\right|\left(\left|P_{0}\right|-1\right),
$$


and hence

$$
\mathbb{E} Z_{0} \leq \mathbb{E}\left[\left|P_{0}\right|\left(\left|P_{0}\right|-1\right)\right]=\frac{1}{2} \lambda_{r}^{2} .
$$

Setting $t=O\left(\log r^{-1}+\log \varepsilon^{-1}\right)$, we obtain $\mathbb{E} Z_{t}<1 / \varepsilon$, and hence $\operatorname{Pr}(T>t) \leq \varepsilon$. It follows that the expected number of iterations of the while-loop of Algorithm 1 is $O\left(\log r^{-1}\right)$. Note that the probability of non-termination decreases exponentially with $t$, so the probability of large deviations above the expected value of $T$ is low.

Crude estimates give $\bar{\lambda}=1 /(4 \sqrt{2})$. The calculation goes as follows. Suppose, in (7), we condition on the random variables BadPoints $\left(P_{0}\right), \ldots$, BadPoints $\left(P_{t}\right)$, rather than simply on $Z_{0}, \ldots, Z_{t}$. This is more stringent conditioning, since the former random variables determine the latter. It is enough to establish (7) under the more stringent conditioning. So fix BadPoints $\left(P_{0}\right)=B_{0}, \ldots$, BadPoints $\left(P_{t}\right)=B_{t}$, and note that this choice also fixes the resampling sets $S_{0}, \ldots, S_{t}$. Suppose $Z_{t}=\left|\operatorname{BadPairs}\left(P_{t}\right)\right|=k$. Inside the resampling set $S_{t}$ we have a Poisson point process $P_{t+1} \cap S_{t}$ of intensity $\lambda_{r}$. Outside, by Lemma 2 , there is a realisation of the hard disks process. Since we are seeking an upper bound on $Z_{t+1}$ we may, by Lemma 3 , replace $P_{t+1} \cap \bar{S}_{t}$ by a Poisson point process of intensity $\lambda_{r}$.

Let $k^{\prime}=\mathbb{E}\left(Z_{t+1} \mid Z_{0}, \ldots, Z_{t}\right)$. From the above considerations we have

$$
k^{\prime} \leq \int_{S_{t}} \lambda_{r} \int_{[0,1]^{2}} \lambda_{r} \mathbf{1}_{\|x-y\| \leq 2 r} d y d x
$$

This is an overestimate, as we are double-counting overlapping disks whose centres both lie within $S_{t}$. Now, $S_{t}$ is a union of at most $2 k$ disks of radius $2 r$. Thus

$$
\begin{aligned}
k^{\prime} & \leq 2 k \lambda_{r}^{2} \int_{D_{2 r}(\mathbf{0})} \int_{\mathbb{R}^{2}} \mathbf{1}_{\|x-y\| \leq 2 r} d y d x \\
& =2 k \lambda_{r}^{2} \int_{D_{2 r}(\mathbf{0})} \int_{D_{2 r}(x)} d y d x \\
& =2 k \lambda_{r}^{2} \times 4 \pi r^{2} \times 4 \pi r^{2} \\
& =32 \lambda^{2} k .
\end{aligned}
$$

There are further sources of slack here: there may be fewer than $2 k$ disks, the disks comprising $S_{t}$ certainly overlap, and, for points $x$ near the boundary, some of disks $D_{2 r}(x)$ will lie partly outside the unit square. (The last of these presumably has no effect asymptotically, as $r \rightarrow 0$.) Setting $\bar{\lambda}=1 /(4 \sqrt{2})=0.17677+$, we see that $\alpha=k / k^{\prime}>1$ for any $\lambda<\bar{\lambda}$, and $\left(Z_{t}\right)_{t=0}^{\infty}$ is a supermartingale, as required.

The constant $\bar{\lambda}$ may seem quite small. Note, however, that classical rejection sampling cannot achieve any $\bar{\lambda}>0$. The argument goes as follows. Divide $[0,1]^{2}$ into $r \times r$ squares. If there are two points in the same square then they will certainly be less than distance $2 r$ apart. The number of points in each square is Poisson distributed with mean $\lambda / \pi$. Thus for any $\lambda>0$ the probability that a particular square has at least two points is bounded away from zero. The number of points in each square is independent of all the others. It follows that the running time of classical rejection sampling is exponential in $r^{-2}$.

The above derivation for $\bar{\lambda}$ is quite crude and can be improved.

- Lemma 6. The constant $\bar{\lambda}$ in Lemma 5 can be taken to be 0.21027 .

Proof. For each of the $2 k$ disks, the right-hand side of inequality (9) counts pairs of points $(x, y)$ with $x$ in the disk, and $y$ anywhere within distance $2 r$ of $x$. Since a bad event is determined by an unordered pair of points, this gives rise to significant double counting. 


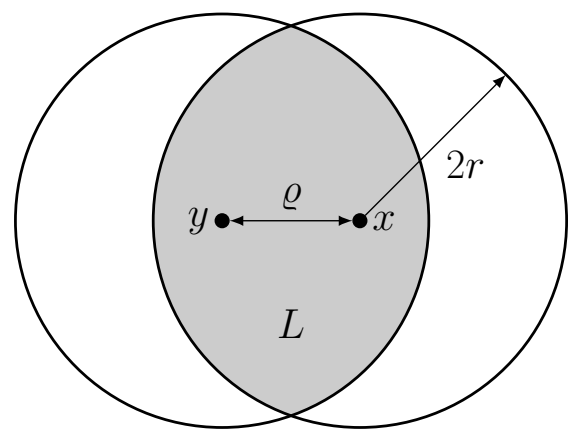

Figure 1 An illustration of double counting.

In particular, pairs $(x, y)$ with $x$ and $y$ lying in the same ball are double counted. We can subtract off these pairs to obtain a better estimate.

For a single ball, the correction is

$$
C=\frac{1}{2} \int_{D_{2 r}(\mathbf{0})} \lambda_{r} \int_{D_{2 r}(\mathbf{0})} \lambda_{r} \mathbf{1}_{\|x-y\| \leq 2 r} d y d x .
$$

(The initial factor of one half arises because we want to count unordered pairs.) With the change of variables $x=2 r x^{\prime}$ and $y=2 r y^{\prime}$ this expression simplifies to

$$
\begin{aligned}
C & =\frac{1}{2} \times 16 r^{4} \lambda_{r}^{2} \int_{D_{1}(\mathbf{0})} \int_{D_{1}(\mathbf{0})} \mathbf{1}_{\left\|x^{\prime}-y^{\prime}\right\| \leq 1} d y^{\prime} d x^{\prime} \\
& =8 \lambda_{r}^{2} r^{4} \int_{D_{1}(\mathbf{0})} L\left(\left\|x^{\prime}\right\|\right) d x^{\prime}
\end{aligned}
$$

where $L\left(\left\|x^{\prime}\right\|\right)$ is the area of the "lens" $D_{1}(\mathbf{0}) \cap D_{1}\left(x^{\prime}\right)$. Letting $\varrho$ denote the offset of the centres of the two disks, the area of the lens is given by

$$
L(\varrho)=2 \arccos (\varrho / 2)-\frac{1}{2} \varrho \sqrt{4-\varrho^{2}} .
$$

(This is by elementary geometry: the lens is the intersection of two sectors, one from each of the disks, and its area can be computed by inclusion-exclusion.) An illustration (before shifting $y$ to $\mathbf{0}$ ) is given in Figure 1 . The shaded area is $L$.

Translating to polar coordinates $(\varrho, \theta)$,

$$
\begin{aligned}
C & =8 \lambda_{r}^{2} r^{4} \int_{D_{1}(\mathbf{0})} L\left(\left\|x^{\prime}\right\|_{2}\right) d x^{\prime} \\
& =8 \lambda_{r}^{2} r^{4} \int_{0}^{2 \pi} \int_{0}^{1} \varrho L(\varrho) d \varrho d \theta \\
& =\frac{8 \lambda^{2}}{\pi^{2}} \times 2 \pi \int_{0}^{1} \varrho L(\varrho) d \varrho \\
& =\frac{16 \lambda^{2}}{\pi}\left[\frac{\pi}{2}+\left(\varrho^{2}-1\right) \arccos \left(\frac{\varrho}{2}\right)-\left(\frac{\varrho}{4}+\frac{\varrho^{3}}{8}\right) \sqrt{4-\varrho^{2}}\right]_{0}^{1} \\
& =\left(8-\frac{6 \sqrt{3}}{\pi}\right) \lambda^{2} .
\end{aligned}
$$

(The integral was evaluated using the Maple computer algebra system.) Our revised upper bound on $k^{\prime}$ is thus

$$
k^{\prime} \leq 2 k\left(16 \lambda^{2}-C\right)=2 k \lambda^{2}\left(8+\frac{6 \sqrt{3}}{\pi}\right),
$$


Solving

$$
\bar{\lambda}^{2}\left(16+\frac{12 \sqrt{3}}{\pi}\right)=1
$$

yields the improved bound of $\bar{\lambda}=0.21027+$.

There are other factors that could in principle be used to increase $\bar{\lambda}$ further - each disk necessarily overlaps with at least one other disk, some bad events are triple or quadruple counted — but the computational difficulties rapidly increase when attempting to account for these.

\section{Three or more dimensions}

In higher dimensions, the hard disk model is known as the hard spheres model. Everything in Sections 3 and 4 carries across to $d>2$ dimensions with little change. For general $d$, the appropriate scaling for the intensity is $\lambda_{r, d}=\lambda /\left(v_{d} r^{d}\right)$, where $v_{d}$ is the volume of a ball of unit radius in $d$ dimensions. Note that in a realisation of a Poisson point process with intensity $\lambda_{r, d}$, the expected number of points in a ball of radius $r$ is $\lambda$.

The analogue of equation (8) is

$$
k^{\prime} \leq \int_{S_{t}} \lambda_{r, d} \int_{[0,1]^{d}} \lambda_{r, d} \mathbf{1}_{\|x-y\| \leq 2 r} d y d x,
$$

which leads to

$$
k^{\prime} \leq 2^{2 d+1} \lambda^{2} k .
$$

So setting $\bar{\lambda}=2^{-\left(d+\frac{1}{2}\right)}$ we find that $\alpha=k / k^{\prime}>1$ for any $\lambda<\bar{\lambda}$. It follows that the running time of partial rejection sampling is $O(\log r)$ for any $\lambda<\bar{\lambda}$.

By a result of Jenssen, Joos and Perkins [6], we lose just a constant factor when translating from intensity $\lambda$ to packing density $\alpha$. (It is partly to connect with their work, we measure intensity in terms of the expected number of points in a ball of radius $r$.) In the proof of $[6$, Thm 2], the following inequality is derived:

$$
\alpha \geq \inf _{z} \max \left\{\lambda e^{-z}, 2^{-d} \exp \left[-2 \cdot 3^{d / 2} \lambda\right] \cdot z\right\} .
$$

Assuming $\lambda \leq \bar{\lambda}$, which holds in the range of validity of our algorithm, we have $\sqrt{2} \lambda \leq 2^{-d}$ and hence

$$
\begin{aligned}
\alpha & \geq \inf _{z} \max \left\{\lambda e^{-z}, \sqrt{2} \lambda \exp \left[-\sqrt{2}(3 / 4)^{d / 2}\right] \cdot z\right\} \\
& =c_{d} \lambda,
\end{aligned}
$$

where

$$
c_{d}=\inf _{z} \max \left\{e^{-z}, \sqrt{2} \exp \left[-\sqrt{2}(3 / 4)^{d / 2}\right] \cdot z\right\} .
$$

Note that $\left(c_{d}\right)$ is monotonically increasing, with $c_{2}=0.42220+$, and $\lim _{d \rightarrow \infty} c_{d}=0.63724+$. It follows that we can reach expected packing density $\Omega\left(2^{-d}\right)$ with $O\left(\log r^{-1}\right)$ expected iterations. This is currently the best that can be achieved by any provably correct sampling algorithm with polynomial (in $1 / r$ ) runtime [7]. The asymptotically best packing density currently rigorously known is $d 2^{-d}$, but achieving this would require $\lambda$ to grow exponentially fast in $d$. This is clearly beyond the capability of partial rejection sampling, but also beyond the capability of any known efficient sampling algorithm. 


\section{References}

1 Henry Cohn. A conceptual breakthrough in sphere packing. Notices Amer. Math. Soc., 64(2):102-115, 2017.

2 Henry Cohn, Abhinav Kumar, Stephen D. Miller, Danylo Radchenko, and Maryna Viazovska. The sphere packing problem in dimension 24. Ann. of Math. (2), 185(3):10171033, 2017.

3 Heng Guo, Mark Jerrum, and Jingcheng Liu. Uniform sampling through the Lovasz local lemma. In STOC, pages 342-355, 2017.

4 Thomas C. Hales. A proof of the Kepler conjecture. Ann. of Math. (2), 162(3):1065-1185, 2005.

5 Thomas P. Hayes and Cristopher Moore. Lower bounds on the critical density in the hard disk model via optimized metrics. CoRR, abs/1407.1930, 2014.

6 Matthew Jenssen, Felix Joos, and Will Perkins. On the hard sphere model and sphere packings in high dimensions. ArXiv, abs/1707.00476, 2017.

7 Ravi Kannan, Michael W. Mahoney, and Ravi Montenegro. Rapid mixing of several Markov chains for a hard-core model. In ISAAC, pages 663-675, 2003.

8 Wilfrid S. Kendall. Perfect simulation for the area-interaction point process. In Probability towards 2000 (New York, 1995), volume 128 of Lect. Notes Stat., pages 218-234. Springer, New York, 1998.

9 Wilfrid S. Kendall and Jesper Møller. Perfect simulation using dominating processes on ordered spaces, with application to locally stable point processes. Adv. Appl. Probab., $32(3): 844-865,2000$.

10 Torgny Lindvall. On Strassen's theorem on stochastic domination. Electron. Comm. Probab., 4:51-59, 1999.

11 Hartmut Löwen. Fun with hard spheres. In Klaus R. Mecke and Dietrich Stoyan, editors, Statistical Physics and Spatial Statistics, pages 295-331, 2000.

12 Nicholas Metropolis, Arianna W. Rosenbluth, Marshall N. Rosenbluth, Augusta H. Teller, and Edward Teller. Equation of state calculations by fast computing machines. J. Chem. Phys., 21(6):1087-1092, 1953.

13 S. B. Moka, S. Juneja, and M. R. H. Mandjes. Perfect sampling for Gibbs processes with a focus on hard-sphere models. ArXiv, abs/1705.00142, 2017.

14 Will Perkins. Birthday inequalities, repulsion, and hard spheres. Proc. Amer. Math. Soc., 144(6):2635-2649, 2016.

15 Chris Preston. Spatial birth-and-death processes (with discussion). Bull. Inst. Internat. Statist., 46(2):371-391, 405-408 (1975), 1975.

16 V. Strassen. The existence of probability measures with given marginals. Ann. Math. Statist., 36:423-439, 1965.

17 David J. Strauss. A model for clustering. Biometrika, 62(2):467-475, 1975.

18 Maryna S. Viazovska. The sphere packing problem in dimension 8. Ann. of Math. (2), 185(3):991-1015, 2017. 